Bull. Korean Math. Soc. 50 (2013), No. 5, pp. 1451-1469

http://dx.doi.org/10.4134/BKMS.2013.50.5.1451

\title{
ON SOME NEW THEOREMS ON MULTIPLIERS \\ IN HARMONIC FUNCTION SPACES IN HIGHER DIMENSION II
}

\author{
Miloš Arsenović and Romi F. Shamoyan
}

\begin{abstract}
We present various new sharp assertions on multipliers in mixed norm, weighted Hardy and new Lizorkin-Triebel spaces of harmonic functions in higher dimension. Some results are new even in onedimensional case.
\end{abstract}

\section{Introduction and preliminaries}

In this paper we continue investigation, started in [3], of spaces of multipliers between certain spaces of harmonic functions on the unit ball. While the subject of multipliers between spaces of analytic functions in the unit disc is a vast one, multipliers between spaces of analytic functions in the unit ball and in the unit polydisc in $\mathbb{C}^{n}$ are less explored, for some results in this direction see [10] and references therein. For results on multipliers between harmonic spaces on the unit disc we refer the reader to [13], where multipliers between harmonic Bergman type classes were considered, and to [9] and [8] for the case of harmonic Hardy classes.

Let us briefly describe the content of the paper. In this section we describe spaces of harmonic functions on the unit ball that are of interest to us and recall definition of multipliers between two such spaces. The next section contains auxiliary results, however some embedding results presented there could be of independent interest. The last section begins with general necessary conditions for a sequence to be a multiplier, these are valid for quite general assumptions on the parameters involved in the definition of the spaces. In many cases these necessary conditions turn out to be sufficient as well and these characterizations of multipliers between various spaces of harmonic functions are the main results of this paper.

Received August 13, 2011.

2010 Mathematics Subject Classification. Primary 30H20.

Key words and phrases. multipliers, spaces of harmonic functions, Bergman type mixed norm spaces, spherical harmonics.

The first author was supported by Ministry of Science, Serbia, project OI174017. 
Let $\mathbb{B}$ be the open unit ball in $\mathbb{R}^{n}, \mathbb{S}=\partial \mathbb{B}$ is the unit sphere in $\mathbb{R}^{n}$, for $x \in \mathbb{R}^{n}$ we have $x=r x^{\prime}$, where $r=|x|=\sqrt{\sum_{j=1}^{n} x_{j}^{2}}$ and $x^{\prime} \in \mathbb{S}$. Let $\omega_{n}$ denote the volume of the unit ball in $\mathbb{R}^{n}$. Normalized Lebesgue measure on $\mathbb{B}$ is denoted by $d x=d x_{1} \cdots d x_{n}=r^{n-1} d r d x^{\prime}$ so that $\int_{\mathbb{B}} d x=1$. We set $I=[0,1)$. We denote the space of all harmonic functions in an open set $\Omega$ by $h(\Omega)$. The gradient of $f \in C^{1}(\Omega)$ is denoted by $\nabla f,|\nabla f(x)|=\sqrt{\sum_{j=1}^{n}\left|\partial f(x) / \partial x_{j}\right|^{2}}$ is its Euclidean norm. In this paper letter $C$ designates a positive constant which can change its value even in the same chain of inequalities.

For $0<p<\infty, 0 \leq r<1$ and $f \in h(\mathbb{B})$ we set

$$
M_{p}(f, r)=\left(\int_{\mathbb{S}}\left|f\left(r x^{\prime}\right)\right|^{p} d x^{\prime}\right)^{1 / p},
$$

with the usual modification to cover the case $p=\infty$. For $0<p<\infty, 0<q \leq$ $\infty, \alpha>0$ and $f \in h(\mathbb{B})$ we consider mixed (quasi)-norms $\|f\|_{p, q, \alpha}$ defined by

$$
\|f\|_{p, q, \alpha}=\left(\int_{0}^{1} M_{q}(f, r)^{p}\left(1-r^{2}\right)^{\alpha p-1} r^{n-1} d r\right)^{1 / p}
$$

and the corresponding spaces

$$
B_{\alpha}^{p, q}(\mathbb{B})=B_{\alpha}^{p, q}=\left\{f \in h(\mathbb{B}):\|f\|_{p, q, \alpha}<\infty\right\} .
$$

For details on these spaces we refer to [4], Chapter 7. In particular these spaces are complete metric spaces and for $\min (p, q) \geq 1$ they are Banach spaces. These mixed norm spaces include weighted Bergman spaces $A_{\beta}^{p}(\mathbb{B})=A_{\beta}^{p}=B_{\frac{\beta+1}{p}}^{p, p}$ where $\beta>-1$ and $0<p<\infty$, see [4] for more on these spaces. We set $A_{\beta}^{\infty}=B_{\beta}^{\infty, \infty}$ for $\beta>0$. We also consider, for $\alpha \geq 0$, weighted Hardy spaces

$$
H_{\alpha}^{p}(\mathbb{B})=H_{\alpha}^{p}=\left\{f \in h(\mathbb{B}):\|f\|_{p, \alpha}=\sup _{r<1} M_{p}(f, r)(1-r)^{\alpha}<\infty\right\}, 0<p \leq \infty .
$$

If $\alpha=0$ we write simply $H^{p}$ for $H_{0}^{p}$. In view of the above definitions it is natural to alow $p=\infty$ in the definition of $B_{\alpha}^{p, q}$ by setting $B_{\alpha}^{\infty, p}=H_{\alpha}^{p}$.

We denote by $B$ the harmonic Bloch space, i.e., the space of all functions $f \in h(\mathbb{B})$ with finite norm

$$
\|f\|_{B}=|f(0)|+\sup _{x \in \mathbb{B}}\left(1-|x|^{2}\right)|\nabla f(x)| .
$$

This space is a Banach space, its closed subspace consisting of all $f \in B$ such that $\lim _{x \rightarrow \mathbb{S}}\left(1-|x|^{2}\right)|\nabla f(x)|=0$ is called a little Bloch space and denoted by $B_{0}$. We refer the reader to [6] for details on $B$ and $B_{0}$.

Analytic Triebel-Lizorkin spaces were studied by several authors, see for example [7], [11] and references therein. In this paper we consider harmonic Triebel-Lizorkin spaces on the unit ball in $\mathbb{R}^{n}$. 
Definition 1. Let $0<p, q<\infty$ and $\alpha>0$. The harmonic Triebel-Lizorkin space $F_{\alpha}^{p, q}(\mathbb{B})=F_{\alpha}^{p, q}$ consists of all functions $f \in h(\mathbb{B})$ such that

$$
\|f\|_{F_{\alpha}^{p, q}}=\left(\int_{\mathbb{S}}\left(\int_{0}^{1}\left|f\left(r x^{\prime}\right)\right|^{p}(1-r)^{\alpha p-1} d r\right)^{q / p} d x^{\prime}\right)^{1 / q}<\infty .
$$

These spaces are complete metric spaces, for $\min (p, q) \geq 1$ they are Banach spaces. We prove certain inclusions between $F_{\alpha}^{p, q}$ and $B_{\alpha}^{p, q}$ spaces (see Propositions 4 and 5).

Next we need certain facts on spherical harmonics, see [14] for a detailed exposition. Let $Y_{j}^{(k)}$ be the spherical harmonics of order $k, j \leq 1 \leq d_{k}$, on $\mathbb{S}$. Next,

$$
Z_{x^{\prime}}^{(k)}\left(y^{\prime}\right)=\sum_{j=1}^{d_{k}} Y_{j}^{(k)}\left(x^{\prime}\right) \overline{Y_{j}^{(k)}\left(y^{\prime}\right)}
$$

are zonal harmonics of order $k$. The spherical harmonics $Y_{j}^{(k)}(k \geq 0,1 \leq j \leq$ $d_{k}$ ) form an orthonormal basis of $L^{2}\left(\mathbb{S}, d x^{\prime}\right)$. Every $f \in h(\mathbb{B})$ has an expansion

$$
f(x)=f\left(r x^{\prime}\right)=\sum_{k=0}^{\infty} r^{k} b_{k} \cdot Y^{k}\left(x^{\prime}\right),
$$

where $b_{k}=\left(b_{k}^{1}, \ldots, b_{k}^{d_{k}}\right), Y^{k}=\left(Y_{1}^{(k)}, \ldots, Y_{d_{k}}^{(k)}\right)$ and $b_{k} \cdot Y^{k}$ is interpreted in the scalar product sense: $b_{k} \cdot Y^{k}=\sum_{j=1}^{d_{k}} b_{k}^{j} Y_{j}^{(k)}$. We often write, to stress dependence on a function $f \in h(\mathbb{B}), b_{k}=b_{k}(f)$ and $b_{k}^{j}=b_{k}^{j}(f)$, in fact we have linear functionals $b_{k}^{j}, k \geq 0,1 \leq j \leq d_{k}$, on the space $h(\mathbb{B})$.

We denote the Poisson kernel for the unit ball by $P\left(x, y^{\prime}\right)$, it is given by

$$
\begin{aligned}
P\left(x, y^{\prime}\right)=P_{y^{\prime}}(x) & =\sum_{k=0}^{\infty} r^{k} \sum_{j=1}^{d_{k}} Y_{j}^{(k)}\left(y^{\prime}\right) Y_{j}^{(k)}\left(x^{\prime}\right) \\
& =\frac{1}{n \omega_{n}} \frac{1-|x|^{2}}{\left|x-y^{\prime}\right|^{n}}, \quad x=r x^{\prime} \in \mathbb{B}, \quad y^{\prime} \in \mathbb{S} .
\end{aligned}
$$

We recall some definitions from [3], these are needed to formulate our main results.

Definition 2. For a double indexed sequence of complex numbers

$$
c=\left\{c_{k}^{j}: k \geq 0,1 \leq j \leq d_{k}\right\}
$$

and a harmonic function $f\left(r x^{\prime}\right)=\sum_{k=0}^{\infty} r^{k} \sum_{j=1}^{d_{k}} b_{k}^{j}(f) Y_{j}^{(k)}\left(x^{\prime}\right)$ we define

$$
(c * f)\left(r x^{\prime}\right)=\sum_{k=0}^{\infty} \sum_{j=1}^{d_{k}} r^{k} c_{k}^{j} b_{k}^{j}(f) Y_{j}^{(k)}\left(x^{\prime}\right), \quad r x^{\prime} \in \mathbb{B},
$$


if the series converges in $\mathbb{B}$. Similarly we define convolution of $f, g \in h(\mathbb{B})$ by

$$
(f * g)\left(r x^{\prime}\right)=\sum_{k=0}^{\infty} \sum_{j=1}^{d_{k}} r^{k} b_{k}^{j}(f) b_{k}^{j}(g) Y_{j}^{(k)}\left(x^{\prime}\right), \quad r x^{\prime} \in \mathbb{B}
$$

it is easily seen that $f * g$ is defined and harmonic in $\mathbb{B}$.

Definition 3. For $t>0$ and a harmonic function $f(x)=\sum_{k=0}^{\infty} r^{k} b_{k}(f) \cdot Y^{k}\left(x^{\prime}\right)$ on $\mathbb{B}$ we define a fractional derivative of order $t$ of $f$ by the following formula:

$$
\left(\Lambda_{t} f\right)(x)=\sum_{k=0}^{\infty} r^{k} \frac{\Gamma(k+n / 2+t)}{\Gamma(k+n / 2) \Gamma(t)} b_{k}(f) \cdot Y^{k}\left(x^{\prime}\right), \quad x=r x^{\prime} \in \mathbb{B} .
$$

Clearly, for $f \in h(\mathbb{B})$ and $t>0$ the function $\Lambda_{t} h$ is also harmonic in $\mathbb{B}$. We also note that $\left(g * P_{y^{\prime}}\right)\left(r x^{\prime}\right)=\left(g * P_{x^{\prime}}\right)\left(r y^{\prime}\right)$ and $\Lambda_{t}(f * g)(x)=\left(\Lambda_{t} f * g\right)(x)$ for any $f, g \in h(\mathbb{B})$, these easy to prove formulae are often used in our proofs without comment.

Definition 4. Let $X$ and $Y$ be subspaces of $h(\mathbb{B})$. We say that a double indexed sequence $c$ is a multiplier from $X$ to $Y$ if $c * f \in Y$ for every $f \in X$. The vector space of all multipliers from $X$ to $Y$ is denoted by $M_{H}(X, Y)$.

Clearly every multiplier $c \in M_{H}(X, Y)$ induces a linear map $M_{c}: X \rightarrow Y$. If, in addition, $X$ and $Y$ are complete (quasi)-normed spaces such that all functionals $b_{k}^{j}$ are continuous on both spaces $X$ and $Y$, then the map $M_{c}$ : $X \rightarrow Y$ is continuous, as is easily seen using the closed graph theorem. This condition is satisfied by all spaces we considered above.

\section{Auxiliary results}

In this section we collect results needed for our main results in the next section. In proving necessary conditions for a double indexed sequence to be a multiplier one uses test functions, these are provided by the Bergman kernel for harmonic weighted Bergman spaces $A_{m}^{p}, m>-1$. This kernel is the following function

(3)

$$
Q_{m}(x, y)=2 \sum_{k=0}^{\infty} \frac{\Gamma(m+1+k+n / 2)}{\Gamma(m+1) \Gamma(k+n / 2)} r^{k} \rho^{k} Z_{x^{\prime}}^{(k)}\left(y^{\prime}\right), \quad x=r x^{\prime}, y=\rho y^{\prime} \in \mathbb{B} .
$$

The test functions we are going to use in the next section are harmonic functions $f_{m, y}(x)=Q_{m}(x, y), y \in \mathbb{B}$. We often write $f_{y}$ instead of $f_{m, y}$.

The following lemma gives an estimate for the kernel $Q_{m}$ (see [4], [5]).

Lemma 1. (a) Let $m>-1$. Then, for $x=r x^{\prime}, y=\rho y^{\prime} \in \mathbb{B}$ we have

$$
\left|Q_{m}(x, y)\right| \leq \frac{C}{\left|\rho x-y^{\prime}\right|^{n+m}}
$$


(b) Let $m>n-1,0 \leq r<1$ and $y^{\prime} \in \mathbb{S}$. Then

$$
\int_{\mathbb{S}} \frac{d x^{\prime}}{\left|r x^{\prime}-y^{\prime}\right|^{m}} \leq \frac{C}{(1-r)^{m-n+1}} .
$$

The following lemma is an often used result from [4].

Lemma 2 ([4]). Let $\alpha>-1$ and $\lambda>\alpha+1$. Then

$$
\int_{0}^{1} \frac{(1-r)^{\alpha}}{(1-r \rho)^{\lambda}} d r \leq C(1-\rho)^{\alpha+1-\lambda}, \quad 0 \leq \rho<1 .
$$

We need some norm estimates of $f_{y}$.

Lemma 3. Let $0<t \leq \infty$. Then we have

$$
M_{t}\left(f_{m, y}, r\right) \leq C(1-r|y|)^{-n-m+\frac{n-1}{t}}, \quad m>\max \left(\frac{n-1}{t}-n,-1\right) .
$$

This follows immediately from Lemma 1 . As a consequence we obtain, using Lemma 2, the following proposition.

Proposition 1. Let $0<p<\infty, 0<t \leq \infty$. Then, for $m>\max \left(\alpha+\frac{n-1}{t}-\right.$ $n,-1)$ we have:

$$
\left\|f_{m, y}\right\|_{B_{\alpha}^{p, t}} \leq C(1-|y|)^{\alpha-n-m+\frac{n-1}{t}}, \quad y \in \mathbb{B} .
$$

The missing case $p=\infty$, i.e., the case of Hardy spaces, is treated in the next proposition.

Proposition 2. Let $0<t \leq \infty, \alpha \geq 0$ and $m>\max \left(\alpha-n+\frac{n-1}{t},-1\right)$. Then we have

$$
\left\|f_{m, y}\right\|_{H_{\alpha}^{t}} \leq C(1-|y|)^{\alpha-n-m+\frac{n-1}{t}}, \quad y \in \mathbb{B} .
$$

The proof of this proposition is similar to the proof of estimate (11) from [3] and can be left to the reader. The following lemma is a preparation for analogous estimates of $F_{\alpha}^{p, t}$ norm of $f_{m, y}$.

Lemma 4. Let $\gamma>-1,0<p<\infty, m>-1$ and $p(n+m)>\gamma+1$. Then we have

(5) $\quad \int_{0}^{1}\left|f_{m, y}\left(r x^{\prime}\right)\right|^{p}(1-r)^{\gamma} d r \leq C\left|x^{\prime}-y\right|^{\gamma+1-p(n+m)}, \quad y \in \mathbb{B}, \quad x^{\prime} \in \mathbb{S}$.

Proof. Using definitions and Lemma 1 we obtain

$$
M=\int_{0}^{1}\left|f_{m, y}\left(r x^{\prime}\right)\right|^{p}(1-r)^{\gamma} d r \leq C \int_{0}^{1} \frac{(1-r)^{\gamma} d r}{\left|x^{\prime}-r y\right|^{p(n+m)}} .
$$

Using elementary geometric inequality $\left|x^{\prime}-r y\right| \geq c\left(\left|x^{\prime}-y\right|+(1-r)\right)$ we obtain, using Lemma 2,

$$
M \leq C \int_{0}^{1} \frac{(1-r)^{\gamma} d r}{\left[\left|x^{\prime}-y\right|+(1-r)\right]^{p(n+m)}} \leq C\left|x^{\prime}-y\right|^{\gamma+1-p(n+m)} .
$$


Proposition 3. Let $0<p, t<\infty$ and $m>\max \left(\alpha+\frac{n-1}{t}-n,-1\right)$. Then we have

$$
\left\|f_{m, y}\right\|_{F_{\alpha}^{p, t}} \leq C(1-|y|)^{\alpha-n-m+\frac{n-1}{t}}, \quad y \in \mathbb{B} .
$$

Proof. Using the above lemma and Lemma 1 we obtain

$$
\begin{aligned}
\left\|f_{m, y}\right\|_{F_{\alpha}^{p, t}}^{t} & =\int_{\mathbb{S}}\left(\int_{0}^{1}\left|f\left(r x^{\prime}\right)\right|^{p}(1-r)^{\alpha p-1} d r\right)^{t / p} d x^{\prime} \\
& \leq C \int_{\mathbb{S}}\left|x^{\prime}-y\right|^{t(\alpha-n-m)} d x^{\prime} \leq C(1-|y|)^{t(\alpha-n-m)+n-1} .
\end{aligned}
$$

The remaining part of this section is devoted to embedding results, these are often used in proofs of our main results in the next section.

Lemma 5. If $0<s \leq t \leq \infty$, then

$$
M_{t}(f, r) \leq C(1-r)^{(n-1)(1 / t-1 / s)} M_{s}(f, r), \quad 0 \leq r<1, \quad f \in h(\mathbb{B}) .
$$

Proof. We can assume $s<t$. Let us set $b=(n-1)\left(\frac{1}{s}-\frac{1}{t}\right)$ and let $I_{b}$ denote the operator of fractional integration of order $b>0$. Now (7) follows immediately from the following estimates:

$$
\begin{aligned}
M_{s}\left(I_{b} f, r\right) & \leq C(1-r)^{-b} M_{s}(f, r), \quad 0 \leq r<1, \\
M_{t}(f, r) & \leq C M_{s}\left(I_{b} f, r\right), \quad 0 \leq r<1 .
\end{aligned}
$$

For the first estimate see [4], Chapter 7, for the second, which is a HardyLittlewood theorem for harmonic functions in the unit ball, see [2], page 47.

If we raise both sides of inequality $(7)$ to the $p$-th power, multiply by (1 $r)^{\alpha p-1}$ and integrate over $I$ we obtain the following corollary.

Corollary 1. Let $0<s \leq t<\infty, 0<p<\infty$ and $\alpha>(n-1)\left(\frac{1}{s}-\frac{1}{t}\right)$. Then

$$
\|f\|_{B_{\alpha}^{p, t}} \leq C\|f\|_{B_{\beta}^{p, s}}, \quad \beta=\alpha+(n-1)(1 / t-1 / s), \quad f \in h(\mathbb{B}),
$$

i.e., $B_{\beta}^{p, s}$ is continuously embedded into $B_{\alpha}^{p, t}$.

The case $p=\infty$ leads us to weighted Hardy spaces, for $0<s \leq t<\infty$ the following result is an immediate consequence of Lemma 5:

$$
\|f\|_{H_{\alpha}^{t}} \leq C\|f\|_{H_{\beta}^{s}}, \quad \beta=\alpha+(n-1)(1 / t-1 / s), \quad f \in h(\mathbb{B}) .
$$

Let us note another continuous embedding from [4]:

$$
B_{\alpha}^{p_{0}, s} \hookrightarrow B_{\alpha}^{p_{1}, s}, \quad 0<s \leq \infty, \quad 0<p_{0} \leq p_{1} \leq \infty, \quad \alpha>0 .
$$

Proposition 4. For $0<t \leq p<\infty$ the space $F_{\alpha}^{p, t}$ is continuously embedded into $B_{\alpha}^{p, t}$ :

$$
\|f\|_{B_{\alpha}^{p, t}} \leq C\|f\|_{F_{\alpha}^{p, t}}, \quad \alpha>0 .
$$


Proof. For $f \in B_{\alpha}^{p, t}$ we have, using continuous form of Minkowski's inequality

$$
\begin{aligned}
\|f\|_{B_{\alpha}^{p, t}}^{p} & \leq C \int_{0}^{1}\left(\int_{\mathbb{S}}\left|f\left(r x^{\prime}\right)\right|^{t} d x^{\prime}\right)^{p / t}(1-r)^{\alpha p-1} d r \\
& =C\left\|(1-r)^{\alpha t} \int_{\mathbb{S}}\left|f\left(r x^{\prime}\right)\right|^{t} d x^{\prime}\right\|_{L^{p / t}\left((1-r)^{-1} d r\right)}^{p / t} \\
& \leq C\left(\int_{\mathbb{S}}\left\|(1-r)^{\alpha t}\left|f\left(r x^{\prime}\right)\right|^{t}\right\|_{L^{p / t}\left((1-r)^{-1} d r\right)} d x^{\prime}\right)^{p / t} \\
& =C\|f\|_{F_{\alpha}^{p, t} .}^{p}
\end{aligned}
$$

Proposition 5. Let $0<p \leq 1$ and $p \leq q$. Then $B_{\alpha}^{p, p}$ is continuously embedded into $F_{\alpha}^{q, p}$ :

$$
\|f\|_{F_{\alpha}^{q, p}} \leq C\|f\|_{B_{\alpha}^{p, p}, \quad f \in h(\mathbb{B})}
$$

Proof. We set $I_{n}=\left[1-2^{-n}, 1-2^{-n-1}\right)$, then we have $\cup_{n=0}^{\infty} I_{n}=I$. Set, for $x^{\prime} \in \mathbb{S}, I_{n}\left(x^{\prime}\right)=\int_{I_{n}}\left|f\left(r x^{\prime}\right)\right|^{q}(1-r)^{\alpha q-1} d r$. Since $p / q \leq 1$ we have

$$
\begin{aligned}
\|f\|_{F_{\alpha}^{q, p}}^{p} & =\int_{\mathbb{S}}\left(\int_{0}^{1}\left|f\left(r x^{\prime}\right)\right|^{q}(1-r)^{\alpha q-1} d r\right)^{p / q} d x^{\prime} \\
& =\int_{\mathbb{S}}\left(\sum_{n=0}^{\infty} I_{n}\left(x^{\prime}\right)\right)^{p / q} d x^{\prime} \leq \int_{\mathbb{S}} \sum_{n=0}^{\infty} I_{n}\left(x^{\prime}\right)^{p / q} d x^{\prime} \\
& =\sum_{n=0}^{\infty} \int_{\mathbb{S}} I_{n}\left(x^{\prime}\right)^{p / q} d x^{\prime}
\end{aligned}
$$

Set $M_{n} f\left(x^{\prime}\right)=\sup _{r \in I_{n}}\left|f\left(r x^{\prime}\right)\right|$ for $n \geq 0$ and $x^{\prime} \in \mathbb{S}$. Then we have (see [2], page 47 )

$$
\int_{\mathbb{S}} M_{n}^{p} f\left(x^{\prime}\right) d x^{\prime} \leq C 2^{n} \int_{I_{n}} M_{p}^{p}(f, r) d r .
$$

Clearly, $I_{n}\left(x^{\prime}\right) \leq C 2^{-n \alpha q} M_{n}^{q} f\left(x^{\prime}\right)$ for $x^{\prime} \in \mathbb{S}$ and therefore we can use the above estimate to get

$$
\begin{aligned}
\|f\|_{F_{\alpha}^{q, p}}^{p} & \leq C \sum_{n=0}^{\infty} \int_{\mathbb{S}} 2^{-n p \alpha} M_{n}^{p} f\left(x^{\prime}\right) d x^{\prime} \leq C \sum_{n=0}^{\infty} 2^{-n(p \alpha-1)} \int_{I_{n}} M_{p}^{p}(f, r) d r \\
& \leq C \sum_{n=0}^{\infty} \int_{I_{n}} M_{p}^{p}(f, r)(1-r)^{\alpha p-1} d r \\
& =\|f\|_{B_{\alpha}^{p, p}} .
\end{aligned}
$$




\section{Multipliers on spaces of harmonic functions}

In this section we present our main results: sufficient and/or necessary conditions for a double indexed sequence $c$ to be in $M_{H}(X, Y)$, for certain (quasi) normed spaces $X$ and $Y$ of harmonic functions. We associate to such a sequence $c$ a harmonic function

$$
g_{c}(x)=g(x)=\sum_{k \geq 0} r^{k} \sum_{j=1}^{d_{k}} c_{k}^{j} Y_{j}^{(k)}\left(x^{\prime}\right), \quad x=r x^{\prime} \in \mathbb{B},
$$

and express our conditions in terms of fractional derivatives of $g_{c}$.

The first part of the lemma below appeared, in dimension two, in [8], for the second part see [3] and [12].

Lemma 6. Let $f, g \in h(\mathbb{B})$ have expansions

$$
f\left(r x^{\prime}\right)=\sum_{k=0}^{\infty} r^{k} \sum_{j=1}^{d_{k}} c_{k}^{j} Y_{j}^{(k)}\left(x^{\prime}\right), \quad g\left(r x^{\prime}\right)=\sum_{l=0}^{\infty} r^{l} \sum_{i=1}^{d_{k}} b_{l}^{i} Y_{i}^{(l)}\left(x^{\prime}\right) .
$$

Then we have

$$
\int_{\mathbb{S}}\left(g * P_{y^{\prime}}\right)\left(r x^{\prime}\right) f\left(\rho x^{\prime}\right) d x^{\prime}=\sum_{k=0}^{\infty} r^{k} \rho^{k} \sum_{j=1}^{d_{k}} b_{k}^{j} c_{k}^{j} Y_{j}^{(k)}\left(y^{\prime}\right), \quad y^{\prime} \in \mathbb{S}, \quad 0 \leq r, \rho<1 .
$$

Moreover, for every $m>-1, y^{\prime} \in \mathbb{S}$ and $0 \leq r, \rho<1$ we have

$$
\begin{aligned}
& \int_{\mathbb{S}}\left(g * P_{y^{\prime}}\right)\left(r x^{\prime}\right) f\left(\rho x^{\prime}\right) d x^{\prime} \\
= & 2 \int_{0}^{1} \int_{\mathbb{S}} \Lambda_{m+1}\left(g * P_{y^{\prime}}\right)\left(r R x^{\prime}\right) f\left(\rho R x^{\prime}\right)\left(1-R^{2}\right)^{m} R^{n-1} d x^{\prime} d R .
\end{aligned}
$$

We note for future use the following formula, contained in Lemma 6 :

$$
(c * f)\left(r^{2} x^{\prime}\right)=\int_{\mathbb{S}}\left(g_{c} * P_{y^{\prime}}\right)\left(r x^{\prime}\right) f\left(r y^{\prime}\right) d y^{\prime}, \quad r \in I, \quad x^{\prime} \in \mathbb{B} .
$$

Also, if $h_{y}=h_{m, y}=M_{c} f_{m, y}$ where $m>-1$ and $y=\rho y^{\prime} \in \mathbb{B}$, then

$$
h_{y}(x)=\sum_{k \geq 0} r^{k} \rho^{k} \sum_{j=1}^{d_{k}} \frac{\Gamma(k+n / 2+m+1)}{\Gamma(k+n / 2) \Gamma(m+1)} c_{k}^{j} Y_{j}^{(k)}\left(y^{\prime}\right) Y_{j}^{(k)}\left(x^{\prime}\right), \quad x=r x^{\prime} \in \mathbb{B} .
$$

This gives the following formula which will be in constant use:

$$
h_{y}(x)=\Lambda_{m+1}\left(g_{c} * P_{y^{\prime}}\right)(\rho x) \quad y=\rho y^{\prime} \in \mathbb{B}, \quad x \in \mathbb{B} .
$$

The first part of the following lemma, which gives necessary conditions for $c$ to be a multiplier, is based on [3].

Lemma 7. Let $0<p, q, t \leq \infty, 1 \leq s \leq \infty$ and $m>\max \left(\alpha+\frac{n-1}{t}-n,-1\right)$. Assume a double indexed sequence $c=\left\{c_{k}^{j}: k \geq 0,1 \leq j \leq d_{k}\right\}$ is a multiplier 
from $B_{\alpha}^{p, t}$ to $B_{\beta}^{q, s}$ and $g=g_{c}$ is defined in (14). Then the following condition is satisfied:

$T_{s}(g)=\sup _{0 \leq \rho<1} \sup _{y^{\prime} \in \mathbb{S}}(1-\rho)^{m-\alpha+\beta+n-\frac{n-1}{t}}\left(\int_{\mathbb{S}}\left|\Lambda_{m+1}\left(g * P_{x^{\prime}}\right)\left(\rho y^{\prime}\right)\right|^{s} d x^{\prime}\right)^{1 / s}<\infty$,

where the case $s=\infty$ requires usual modification.

Also, let $0<p, t \leq \infty, 0<s \leq \infty, \alpha>0, \beta \geq 0$ and $m>\max \left(\alpha+\frac{n-1}{t}-\right.$ $n,-1)$. If a double indexed sequence $c=\left\{c_{k}^{j}: k \geq 0,1 \leq j \leq d_{k}\right\}$ is a multiplier from $B_{\alpha}^{p, t}$ to $H_{\beta}^{s}$, then the above function $g$ satisfies condition (17).

Proof. Let $c \in M_{H}\left(B_{\alpha}^{p, t}, B_{\beta}^{q, s}\right)$, and assume both $p$ and $q$ are finite, the infinite cases require only small modifications. We have $\left\|M_{c} f\right\|_{B_{\beta}^{q, s}} \leq C\|f\|_{B_{\alpha}^{p, t}}$ for $f$ in $B_{\alpha}^{p, t}$. Set $h_{y}=M_{c} f_{y}$, then we have

$$
\left\|h_{y}\right\|_{B_{\beta}^{q, s}} \leq C\left\|f_{y}\right\|_{B_{\alpha}^{p, t}}
$$

This estimate and Proposition 1 give

$$
\left\|h_{y}\right\|_{B_{\beta}^{q, s}} \leq C(1-|y|)^{\alpha-m-n+\frac{n-1}{t}}, \quad y \in \mathbb{B} .
$$

Using (16) and monotonicity of $M_{s}\left(h_{y}, r\right)$ we obtain, for $y=\rho y^{\prime} \in \mathbb{B}$ :

$$
\begin{aligned}
M_{s}\left(\Lambda_{m+1}\left(g * P_{y^{\prime}}\right), \rho^{2}\right)= & \left(\int_{\rho}^{1}(1-r)^{\beta q-1} r^{n-1} d r\right)^{-1 / q} \\
& \times\left(\int_{\rho}^{1}(1-r)^{\beta q-1} r^{n-1} M_{s}^{q}\left(h_{y}, \rho^{2}\right) d r\right)^{1 / q} \\
\leq & C(1-\rho)^{-\beta}\left(\int_{\rho}^{1}(1-r)^{\beta q-1} r^{n-1} M_{s}^{q}\left(h_{y}, r\right) d r\right)^{1 / q} \\
\leq & C(1-\rho)^{-\beta}\left\|h_{y}\right\|_{B_{\beta}^{q, s}}
\end{aligned}
$$

Combining (20) and (19) we obtain

$$
\left(\int_{\mathbb{S}}\left|\Lambda_{m+1}\left(g * P_{x^{\prime}}\right)\left(\rho^{2} y^{\prime}\right)\right|^{s} d x^{\prime}\right)^{1 / s} \leq C(1-\rho)^{\alpha-\beta-m-n+\frac{n-1}{t}},
$$

which is equivalent to (17). The case $s=\infty$ is treated similarly.

Next we consider $c \in M_{H}\left(B_{\alpha}^{p, t}, H_{\beta}^{s}\right)$, assuming $0<p \leq \infty$. Set $h_{y}=M_{c} f_{y}=$ $g * f_{y}$. We have, by Proposition 1 ,

$$
\left\|f_{y}\right\|_{B_{\alpha}^{p, t}} \leq C(1-|y|)^{\alpha-m-n+\frac{n-1}{t}}, \quad y \in \mathbb{B},
$$

and, by continuity of $M_{c},\left\|h_{y}\right\|_{H_{\beta}^{s}} \leq C\left\|f_{y}\right\|_{B_{\alpha}^{p, t}}$. Therefore

$$
\left\|h_{y}\right\|_{H_{\beta}^{s}} \leq C(1-|y|)^{\alpha-m-n+\frac{n-1}{t}}, \quad y \in \mathbb{B} .
$$


Setting $y=\rho y^{\prime}$ we have

$$
\begin{aligned}
I_{y^{\prime}}\left(\rho^{2}\right) & =\left(\int_{\mathbb{S}}\left|\Lambda_{m+1}\left(g * P_{x^{\prime}}\right)\left(\rho^{2} y^{\prime}\right)\right|^{s} d x^{\prime}\right)^{1 / s} \\
& =\left(\int_{\mathbb{S}}\left|\Lambda_{m+1}\left(g * P_{y}\right)\left(\rho x^{\prime}\right)\right|^{s} d x^{\prime}\right)^{1 / s} \\
& =M_{s}\left(h_{y}, \rho\right) \leq(1-|y|)^{-\beta}\left\|h_{y}\right\|_{H_{\beta}^{s}} .
\end{aligned}
$$

The last two estimates yield

$$
\left(\int_{\mathbb{S}}\left|\Lambda_{m+1}\left(g * P_{x^{\prime}}\right)\left(\rho^{2} y^{\prime}\right)\right|^{s} d x^{\prime}\right)^{1 / s} \leq C(1-|y|)^{\alpha-\beta-m-n+\frac{n-1}{t}}, \quad|y|=\rho
$$

which is equivalent to (17).

The first part of the above lemma combined with Proposition 4 gives the following corollary.

Corollary 2. If $c \in M_{H}\left(B_{\alpha}^{p, t}, F_{\beta}^{q, s}\right)$, where $0<p, t \leq \infty, 1 \leq s \leq q<\infty$ and $m>\max \left(\alpha+\frac{n-1}{t}-n,-1\right)$, then the function $g_{c}$ satisfies condition

$$
T_{s}(g)=\sup _{0 \leq \rho<1} \sup _{y^{\prime} \in \mathbb{S}}(1-\rho)^{\beta-\alpha+m+n-\frac{n-1}{t}}\left(\int_{\mathbb{S}}\left|\Lambda_{m+1}\left(g * P_{x^{\prime}}\right)\left(\rho y^{\prime}\right)\right|^{s} d x^{\prime}\right)^{1 / s}<\infty .
$$

Lemma 8. Let $0<p, t<\infty, 0<q \leq \infty, 1 \leq s \leq \infty$, and $m>\max (\alpha+$ $\left.\frac{n-1}{t}-n,-1\right)$. Assume $c \in M_{H}\left(F_{\alpha}^{p, t}, B_{\beta}^{q, s}\right)$. Then the function $g=g_{c}$ satisfies the following condition:

$T_{s}(g)=\sup _{0 \leq \rho<1} \sup _{y^{\prime} \in \mathbb{S}}(1-\rho)^{m+\beta-\alpha+n-\frac{n-1}{t}}\left(\int_{\mathbb{S}}\left|\Lambda_{m+1}\left(g * P_{x^{\prime}}\right)\left(\rho y^{\prime}\right)\right|^{s} d x^{\prime}\right)^{1 / s}<\infty$.

Next, if $c \in M_{H}\left(F_{\alpha}^{p, t}, H_{\beta}^{s}\right)$, where $0<p, t<\infty, 0<s \leq \infty, \alpha>0, \beta \geq 0$ and $m>\max \left(\alpha+\frac{n-1}{t}-n,-1\right)$, then $g=g_{c}$ satisfies condition (24).

Finally, if $c \in M_{H}\left(H_{\alpha}^{t}, H_{\beta}^{s}\right)$, where $m>\max \left(\alpha+\frac{n-1}{t}-n,-1\right), 0<s, t \leq \infty$ and $\alpha \geq 0, \beta \geq 0$, then again $g=g_{c}$ satisfies condition (24).

Proofs of all three statements are analogous to the proof of Lemma 7. For the first one use (6) and (20), for the second one use (6) and (23) and for the last one use (4) and (23). We leave details to the reader.

In our previous work all characterizations of multipliers were independent on the dimension of the space. However, in the following theorem dimension of the space is present in the description of the space of multipliers.

Theorem 1. Let $0<t, p \leq 1,1 \leq s \leq \infty$ and $m>\max \left(\alpha+\frac{n-1}{t}-n,-1\right)$. Then for a double indexed sequence $c=\left\{c_{k}^{j}: k \geq 0,1 \leq j \leq d_{k}\right\}$ the following conditions are equivalent:

1. $c \in M_{H}\left(B_{\alpha}^{p, t}, H_{\beta}^{s}\right)$. 
2. The function $g(x)=\sum_{k \geq 0} r^{k} \sum_{j=1}^{d_{k}} c_{k}^{j} Y_{j}^{(k)}\left(x^{\prime}\right)$ is harmonic in $\mathbb{B}$ and satisfies the following condition

$T_{s}(g)=\sup _{0 \leq \rho<1} \sup _{y^{\prime} \in \mathbb{S}}(1-\rho)^{\beta-\alpha+m+n-\frac{n-1}{t}}\left(\int_{\mathbb{S}}\left|\Lambda_{m+1}\left(g * P_{x^{\prime}}\right)\left(\rho y^{\prime}\right)\right|^{s} d x^{\prime}\right)^{1 / s}<\infty$.

For $1<t \leq \infty$ condition 1 implies condition 2 .

Proof. The necessity of the condition (25) is contained in Lemma 7. Now we prove sufficiency of condition (25). Let $f \in B_{\alpha}^{p, t}$ and set $h=M_{c} f$. We have, by Lemma 6 :

$$
h\left(r^{2} x^{\prime}\right)=2 \int_{0}^{1} \int_{\mathbb{S}} \Lambda_{m+1}\left(g * P_{\xi}\right)\left(r R x^{\prime}\right) f(r R \xi)\left(1-R^{2}\right)^{m} R^{n-1} d \xi d R .
$$

Therefore, since $s \geq 1$, we deduce

$$
M_{s}\left(h, r^{2}\right) \leq C T_{s}(g) \int_{0}^{1}(1-R)^{m} M_{1}(f, r R)(1-r R)^{\alpha-\beta-m-n+\frac{n-1}{t}} R^{n-1} d R .
$$

Now we use Lemma 3 from [3] and obtain

$$
\begin{aligned}
M_{s}^{p}\left(h, r^{2}\right) & \leq C T_{s}^{p}(g) \int_{0}^{1} \frac{(1-R)^{m p+p-1}}{(1-r R)^{p(m+n+\beta-\alpha)-\frac{p}{t}(n-1)}} M_{1}^{p}(f, r R) R^{n-1} d R \\
& \leq C T_{s}^{p}(g)(1-r)^{-\beta p} \int_{0}^{1} \frac{(1-R)^{m p+p-1}}{(1-r R)^{p(m+n-\alpha)-p \frac{n-1}{t}}} M_{1}^{p}(f, r R) R^{n-1} d R .
\end{aligned}
$$

This inequality, monotonicity of $M_{1}(f, r)$ and Lemma 5 give

$$
\begin{aligned}
(1-r)^{\beta p} M_{s}^{p}\left(h, r^{2}\right) & \leq C T_{s}^{p}(g) \int_{0}^{1} \frac{(1-R)^{m p+p-1}}{(1-r R)^{p(m+n-\alpha)-p \frac{n-1}{t}}} M_{1}^{p}(f, R) R^{n-1} d R \\
& \leq C T_{s}^{p}(g) \int_{0}^{1}(1-R)^{p-1+p\left(\frac{n-1}{t}+\alpha-n\right)} M_{1}^{p}(f, R) d R \\
& \leq C T_{s}^{p}(g) \int_{0}^{1}(1-R)^{\alpha p-1} M_{t}^{p}(f, R) R^{n-1} d R \\
& =C T_{s}^{p}(g)\|f\|_{B_{\alpha}^{p, t}}^{p}
\end{aligned}
$$

which implies $\|h\|_{H_{\beta}^{s}} \leq C T_{s}(g)\|f\|_{B_{\alpha}^{p, t}}$ and the proof is complete.

Theorem 1, Lemma 8 and Proposition 4 combine to give the following result.

Theorem 2. Let $0<t \leq p \leq 1 \leq s \leq \infty$ and $m>\max \left(\alpha+\frac{n-1}{t}-n,-1\right)$. Then for a double indexed sequence $c=\left\{c_{k}^{j}: k \geq 0,1 \leq j \leq d_{k}\right\}$ the following conditions are equivalent:

1. $c \in M_{H}\left(F_{\alpha}^{p, t}, H_{\beta}^{s}\right)$. 
2. The function $g(x)=\sum_{k \geq 0} r^{k} \sum_{j=1}^{d_{k}} c_{k}^{j} Y_{j}^{(k)}\left(x^{\prime}\right)$ is harmonic in $\mathbb{B}$ and satisfies the following condition

$T_{s}(g)=\sup _{0 \leq \rho<1} \sup _{y^{\prime} \in \mathbb{S}}(1-\rho)^{\beta-\alpha+m+n-\frac{n-1}{t}}\left(\int_{\mathbb{S}}\left|\Lambda_{m+1}\left(g * P_{x^{\prime}}\right)\left(\rho y^{\prime}\right)\right|^{s} d x^{\prime}\right)^{1 / s}<\infty$.

A characterization of $M_{H}\left(H_{\alpha}^{1}, H_{\beta}^{s}\right), 1 \leq s \leq \infty$, was given in [3], the next two theorems generalize that result.

Theorem 3. Let $0<t \leq 1 \leq s \leq \infty, \alpha \geq 0, m>\max \left(\alpha+\frac{n-1}{t}-n,-1\right)$ and $\beta>0$. Then for a double indexed sequence $c=\left\{c_{k}^{j}: k \geq 0,1 \leq j \leq d_{k}\right\}$ the following conditions are equivalent:

1. $c \in M_{H}\left(H_{\alpha}^{t}, H_{\beta}^{s}\right)$.

2. The function $g(x)=\sum_{k \geq 0} r^{k} \sum_{j=1}^{d_{k}} c_{k}^{j} Y_{j}^{(k)}\left(x^{\prime}\right)$ is harmonic in $\mathbb{B}$ and satisfies the following condition

$T_{s}(g)=\sup _{0 \leq \rho<1} \sup _{y^{\prime} \in \mathbb{S}}(1-\rho)^{\beta-\alpha+m+n-\frac{n-1}{t}}\left(\int_{\mathbb{S}}\left|\Lambda_{m+1}\left(g * P_{x^{\prime}}\right)\left(\rho y^{\prime}\right)\right|^{s} d x^{\prime}\right)^{1 / s}<\infty$.

Proof. The necessity of (26) is contained in Lemma 8. Let us prove sufficiency. We choose $f \in H_{\alpha}^{t}$ and set $h=M_{c} f$. Applying the operator $\Lambda_{m+1}$ to equation (15) we obtain $\Lambda_{m+1} h(r x)=\int_{\mathbb{S}} \Lambda_{m+1}\left(g * P_{y^{\prime}}\right)(x) f\left(r y^{\prime}\right) d y^{\prime}$. Since $s \geq 1$ this gives:

$$
\begin{aligned}
M_{s}\left(\Lambda_{m+1} h, r^{2}\right) & \leq M_{1}(f, r) \sup _{y^{\prime} \in \mathbb{S}}\left\|\Lambda_{m+1}\left(g * P_{y^{\prime}}\right)(x)\right\|_{L^{s}\left(d x^{\prime}\right)} \\
& \leq T_{s}(g)(1-r)^{\alpha-\beta-m-n+\frac{n-1}{t}} M_{1}(f, r) \\
& \leq T_{s}(g)(1-r)^{\alpha-\beta-m-1} M_{t}(f, r),
\end{aligned}
$$

where we at the last step used Lemma 5 . Hence we obtained

$$
(1-r)^{m+1+\beta} M_{s}\left(\Lambda_{m+1} h, r^{2}\right) \leq C(1-r)^{\alpha} M_{t}(f, r), \quad 0 \leq r<1,
$$

and, since $\beta>0$, this implies $(1-r)^{\beta} M_{s}\left(h, r^{2}\right) \leq C\|f\|_{H_{\alpha}^{t}}$ (see [4, Chapter 7]). Hence $\|h\|_{H_{\beta}^{s}} \leq C\|f\|_{H_{\alpha}^{t}}$.

We note that the above proof of sufficiency does not work in the case $\beta=0$. In the following theorem we deal with unweighted Hardy spaces.

Theorem 4. Let $0<t<1 \leq s \leq \infty$ and $m>\max \left(\frac{n-1}{t}-n,-1\right)$. Then for $a$ double indexed sequence $c=\left\{c_{k}^{j}: k \geq 0,1 \leq j \leq d_{k}\right\}$ the following conditions are equivalent:

1. $c \in M_{H}\left(H^{t}, H^{s}\right)$.

2. The function $g(x)=\sum_{k \geq 0} r^{k} \sum_{j=1}^{d_{k}} c_{k}^{j} Y_{j}^{(k)}\left(x^{\prime}\right)$ is harmonic in $\mathbb{B}$ and satisfies the following condition

$$
T_{s}(g)=\sup _{0 \leq \rho<1} \sup _{y^{\prime} \in \mathbb{S}}(1-\rho)^{m+n-\frac{n-1}{t}}\left(\int_{\mathbb{S}}\left|\Lambda_{m+1}\left(g * P_{x^{\prime}}\right)\left(\rho y^{\prime}\right)\right|^{s} d x^{\prime}\right)^{1 / s}<\infty .
$$


Proof. As in the previous theorem, necessity of condition (27) follows from Lemma 8. Let $g=g_{c}$ satisfy (27), let $f \in H_{\alpha}^{t}$ and set $h=M_{c} f$. Then, using Lemma 6 and continuous form of Minkowski's inequality we obtain:

$$
\begin{aligned}
M_{s}\left(h, r^{2}\right) & \leq C \int_{0}^{1} M_{s}\left(\Lambda_{m+1}\left(g * P_{y^{\prime}}\right), r R\right) \int_{\mathbb{S}}\left|f\left(r R x^{\prime}\right)\right| d x^{\prime}(1-R)^{m} R^{n-1} d R \\
& \leq C T_{s}(g) \int_{0}^{1}(1-r R)^{-m-n+\frac{n-1}{t}}(1-R)^{m} M_{1}(f, r R) d R \\
& \leq C T_{s}(g) \int_{0}^{1}(1-R)^{-n+\frac{n-1}{t}} M_{1}(f, R) d R \\
& \leq C T_{s}(g)\|f\|_{H^{t}},
\end{aligned}
$$

the last estimate is a corollary of Carleson-Duren embedding theorem (see [1]).

The theorem below is the first result on multipliers into Triebel-Lizorkin spaces.

Theorem 5. Let $0<p \leq 1 \leq q \leq \infty$ and $m>\alpha-1$. Then for a double indexed sequence $c=\left\{c_{k}^{j}: k \geq 0,1 \leq j \leq d_{k}\right\}$ the following conditions are equivalent:

1. $c \in M_{H}\left(B_{\alpha}^{p, 1}, F_{\beta}^{q, 1}\right)$.

2. The function $g(x)=\sum_{k \geq 0} r^{k} \sum_{j=1}^{d_{k}} c_{k}^{j} Y_{j}^{(k)}\left(x^{\prime}\right)$ is harmonic in $\mathbb{B}$ and satisfies the following condition

$$
N_{1}(g)=\sup _{0 \leq \rho<1} \sup _{y^{\prime} \in \mathbb{S}}(1-\rho)^{\beta-\alpha+m+1} \int_{\mathbb{S}}\left|\Lambda_{m+1}\left(g * P_{x^{\prime}}\right)\left(\rho y^{\prime}\right)\right| d x^{\prime}<\infty .
$$

Proof. Necessity of condition (28) is contained in Corollary 2. Now we choose $c$ such that the condition (28) is satisfied. Then, by Theorem 6 from [3] we have $M_{c}: B_{\alpha}^{p, 1} \rightarrow B_{\beta}^{1,1}$. Since, by Proposition $5, B_{\beta}^{1,1} \hookrightarrow F_{\beta}^{q, 1}$ the proof is complete.

The following theorem is a generalization of Theorem 3 from [3]. The proof is included for reader's convenience, it follows almost the same pattern as in $[3]$.

Theorem 6. Let $1 \leq p \leq q \leq \infty, 1 \leq s \leq \infty$ and $m>\alpha-1$. Then for $a$ double indexed sequence $c=\left\{c_{k}^{j}: k \geq 0,1 \leq j \leq d_{k}\right\}$ the following conditions are equivalent:

1. $c \in M_{H}\left(B_{\alpha}^{p, 1}, B_{\beta}^{q, s}\right)$.

2. The function $g(x)=\sum_{k \geq 0} r^{k} \sum_{j=1}^{d_{k}} c_{k}^{j} Y_{j}^{(k)}\left(x^{\prime}\right)$ is harmonic in $\mathbb{B}$ and satisfies the following condition

$$
N_{s}(g)=\sup _{0 \leq \rho<1} \sup _{y^{\prime} \in \mathbb{S}}(1-\rho)^{\beta-\alpha+m+1}\left(\int_{\mathbb{S}}\left|\Lambda_{m+1}\left(g * P_{x^{\prime}}\right)\left(\rho y^{\prime}\right)\right|^{s} d x^{\prime}\right)^{1 / s}<\infty .
$$


Proof. Since necessity of (29) is contained in Lemma 7 we prove sufficiency of condition (29). We assume $p$ and $q$ are finite, the remaining cases can be treated in a similar manner. Take $f \in B_{\alpha}^{p, 1}$ and set $h=M_{c} f$. Applying the operator $\Lambda_{m+1}$ to both sides of equation (15) we obtain

$$
\Lambda_{m+1} h(r x)=\int_{\mathbb{S}} \Lambda_{m+1}\left(g * P_{y^{\prime}}\right)(x) f\left(r y^{\prime}\right) d y^{\prime}
$$

Now we estimate the $L^{s}$ norm of the above function on $|x|=r$ :

$$
\begin{aligned}
M_{s}\left(\Lambda_{m+1} h, r^{2}\right) & \leq \int_{\mathbb{S}} M_{s}\left(\Lambda_{m+1}\left(g * P_{y^{\prime}}\right), r\right)\left|f\left(r y^{\prime}\right)\right| d y^{\prime} \\
& \leq M_{1}(f, r) \sup _{y^{\prime} \in \mathbb{S}}\left(\int_{\mathbb{S}}\left|\Lambda_{m+1}\left(g * P_{y^{\prime}}\right)\left(r x^{\prime}\right)\right|^{s} d x^{\prime}\right)^{1 / s} \\
& \leq M_{1}(f, r) N_{s}(g)(1-r)^{\alpha-\beta-m-1} .
\end{aligned}
$$

Since,

$$
\begin{aligned}
& \int_{0}^{1} M_{s}^{p}\left(h, r^{2}\right)(1-r)^{\beta p-1} r^{n-1} d r \\
\leq & C \int_{0}^{1}(1-r)^{p(m+1)} M_{s}^{p}\left(\Lambda_{m+1} h, r^{2}\right)(1-r)^{\beta p-1} r^{n-1} d r
\end{aligned}
$$

(see [4]), we have

$$
\begin{aligned}
\|h\|_{B_{\beta}^{p, s}}^{p} & \leq C \int_{0}^{1}(1-r)^{p(m+1)} M_{s}^{p}\left(\Lambda_{m+1} h, r^{2}\right)(1-r)^{\beta p-1} r^{n-1} d r \\
& \leq C N_{s}^{p}(g) \int_{0}^{1} M_{1}^{p}(f, r)(1-r)^{\alpha p-1} r^{n-1} d r \\
& =C N_{s}^{p}(g)\|f\|_{B_{\alpha}^{p, 1}}^{p}
\end{aligned}
$$

and therefore $\|h\|_{B_{\beta}^{p, s}} \leq\|f\|_{B_{\alpha}^{p, 1}}$. Since $\|h\|_{B_{\beta}^{q, s}} \leq C\|h\|_{B_{\beta}^{p, s}}$, see (12), the proof is complete.

Next we develop another approach to multiplier problems in harmonic function spaces that hinges upon duality results. The first of these duality results is from [15]: $\left(B_{\alpha}^{p, q}\right)^{*} \simeq B_{\alpha}^{p^{\prime}, q^{\prime}}$, where $1<p<\infty, 1 \leq q \leq \infty$ and $p^{\prime}$ (resp. $q^{\prime}$ ) is the exponent conjugate to $p$ (resp. $q$ ). Namely, the above identification of the dual space is with respect to the pairing

$$
\langle f, g\rangle=\int_{\mathbb{B}} f(x) g(x)\left(1-|x|^{2}\right)^{2 \alpha-1} d x, \quad f \in B_{\alpha}^{p, q}, \quad g \in B_{\alpha}^{p^{\prime}, q^{\prime}} .
$$

In particular, the spaces $B_{\alpha}^{p, q}$ are reflexive for $1<p<\infty, 1 \leq q \leq \infty$. One can easily verify that for $c \in M_{H}\left(B_{\alpha}^{p_{1}, q_{1}}, B_{\alpha}^{p_{2}, q_{2}}\right)$, where $1<p_{1}, p_{2}<\infty$, $1 \leq q_{1}, q_{2} \leq \infty$, the adjoint operator $M_{c}^{*}: B_{\alpha}^{p_{2}^{\prime}, q_{2}^{\prime}} \rightarrow B_{\alpha}^{p_{1}^{\prime}, q_{1}^{\prime}}$ is also a multiplier operator generated by $c$. 
Theorem 7. Let $1<p, q<\infty$ and $m>\alpha-1$. Then for a double indexed sequence $c=\left\{c_{k}^{j}: k \geq 0,1 \leq j \leq d_{k}\right\}$ the following conditions are equivalent:

1. $c \in M_{H}\left(B_{\alpha}^{p, \infty}, B_{\alpha}^{q, \infty}\right)$.

2. The function $g(x)=\sum_{k \geq 0} r^{k} \sum_{j=1}^{d_{k}} c_{k}^{j} Y_{j}^{(k)}\left(x^{\prime}\right)$ is harmonic in $\mathbb{B}$ and satisfies the following condition

$$
N_{1}(g)<\infty .
$$

Proof. Assume $c \in M_{H}\left(B_{\alpha}^{p, \infty}, B_{\alpha}^{q, \infty}\right)$, then $M_{c}$ maps $B_{\alpha}^{p, \infty}$ continuously into $B_{\alpha}^{q, \infty}$ and therefore $M_{c}^{*}=M_{c}$ maps $B_{\alpha}^{q^{\prime}, 1}$ into $B_{\alpha}^{p^{\prime}, 1}$. Hence, by Theorem 6 , condition (32) is satisfied. Conversely, assume (32) is satisfied. Then, by Theorem $6, M_{c}$ maps $B_{\alpha}^{q^{\prime}, 1}$ into $B_{\alpha}^{p^{\prime}, 1}$. Therefore, $M_{c}^{*}=M_{c} \operatorname{maps}\left(B_{\alpha}^{p, \infty}\right)^{* *}=$ $B_{\alpha}^{p, \infty}$ continuously into $\left(B_{\alpha}^{q, \infty}\right)^{* *}=B_{\alpha}^{q, \infty}$, and the proof is complete.

Now we recall a duality result from [5].

Proposition 6. Let $1<p<\infty, \alpha>0$ and let $q$ be the exponent conjugate to p. Then $\left(A_{\alpha}^{p}\right)^{*} \simeq A_{\alpha}^{q}$ with respect to the pairing

$$
\langle u, v\rangle=\int_{\mathbb{B}} u(x) v(x)\left(1-|x|^{2}\right)^{\alpha} d x .
$$

Again, it is easy to see that if we have a multiplier $M_{c}: A_{\alpha}^{p_{1}} \rightarrow A_{\alpha}^{p_{2}}$, where $1<p_{1}, p_{2}<\infty$, then the adjoint operator $M_{c}^{*}: A_{\alpha}^{p_{2}^{\prime}} \rightarrow A_{\alpha}^{p_{1}^{\prime}}$ is also a multiplier operator generated by the same sequence $c$. Therefore we have the following proposition.

Proposition 7. $M_{H}\left(A_{\alpha}^{p_{1}}, A_{\alpha}^{p_{2}}\right)=M_{H}\left(A_{\alpha}^{p_{2}^{\prime}}, A_{\alpha}^{p_{1}^{\prime}}\right), 1<p_{1}, p_{2}<\infty, \alpha>0$.

The following duality result is also from [5].

Proposition 8. For $\alpha>-1$ we have $B_{0}^{*} \simeq A_{\alpha}^{1}$ and $\left(A_{\alpha}^{1}\right)^{*} \simeq B$. Both identifications are with respect to the following pairing:

$$
\langle f, g\rangle=\int_{\mathbb{B}} f(x) g(x)\left(1-|x|^{2}\right)^{\alpha} d x .
$$

Note that for given $g \in B$ integral in (33) is not necessarily convergent for all $f \in A_{\alpha}^{1}$, however, it converges on a dense subset $A_{\alpha}^{2}$ and extends by continuity to $A_{\alpha}^{1}$, see [5] for details. Since in this situation we again have $M_{c}^{*}=M_{c}$ one immediately obtains

$$
M_{H}\left(B_{0}, B_{0}\right) \subset M_{H}\left(A_{\alpha}^{1}, A_{\alpha}^{1}\right) \subset M_{H}(B, B), \quad \alpha>-1 .
$$

This allows us to characterize multipliers from $B_{0}$ to $B_{0}$.

Theorem 8. Let $m>-1$. For a double indexed sequence $c=\left\{c_{k}^{j}: k \geq 0,1 \leq\right.$ $\left.j \leq d_{k}\right\}$ the following conditions are equivalent:

1. $c \in M_{H}\left(B_{0}, B_{0}\right)$. 
2. The function $g(x)=\sum_{k \geq 0} r^{k} \sum_{j=1}^{d_{k}} c_{k}^{j} Y_{j}^{(k)}\left(x^{\prime}\right)$ is harmonic in $\mathbb{B}$ and satisfies the following condition:

$$
\tilde{N}_{1}(g)=\sup _{0 \leq \rho<1} \sup _{y^{\prime} \in \mathbb{S}}(1-\rho)^{m+1} \int_{\mathbb{S}}\left|\Lambda_{m+1}\left(g * P_{x^{\prime}}\right)\left(\rho y^{\prime}\right)\right| d x^{\prime}<\infty .
$$

Proof. Since $A_{\alpha}^{1}=B_{\alpha+1}^{1,1}$, Theorem 6 and (34) show that condition (35) is necessary for $c \in M_{H}\left(B_{0}, B_{0}\right)$. Now we assume $g_{c}=g$ satisfies (35) and choose $f \in B_{0}$. Set $h=M_{c} f$. Since $\Lambda_{m+1} \nabla=\nabla \Lambda_{m+1}$ on $h(\mathbb{B})$ we obtain, for $0 \leq r<1$ :

The proof is going to rely on the following easily checked identity

$$
\int_{\mathbb{S}} \nabla^{-1} G(y) \nabla g(y) d y=\int_{\mathbb{S}} G(y) g(y) d y,
$$

where $G: \mathbb{B} \rightarrow \mathbb{C}^{n}$ is a gradient of a harmonic function and $g \in h(\mathbb{B})$.

Now we have

$$
\begin{aligned}
\Lambda_{m+1} \nabla h(r x) & =\nabla \Lambda_{m+1} h(r x)=\int_{\mathbb{S}} \nabla_{x}\left(\Lambda_{m+1}\left(g * P_{y^{\prime}}\right)(x) f\left(r y^{\prime}\right) d y^{\prime}\right. \\
& =\int_{\mathbb{S}} \nabla_{y}^{-1} \nabla_{x}\left(\Lambda_{m+1}\left(g * P_{y^{\prime}}\right)(x) \nabla_{y} f(y) d y^{\prime} .\right.
\end{aligned}
$$

Therefore

$$
\begin{aligned}
\left|\Lambda_{m+1} \nabla h(r x)\right| & \leq(1-r)^{-1}\|f\|_{B_{0}} \int_{\mathbb{S}}\left|\Lambda_{m+1} \nabla_{y}^{-1} \nabla_{x}\left(g * P_{y^{\prime}}\right)\left(r x^{\prime}\right)\right| d y^{\prime} \\
& =(1-r)^{-1}\|f\|_{B_{0}} \int_{\mathbb{S}}\left|\Lambda_{m+1} \nabla_{y}^{-1}\left(g * \nabla_{x} P_{x^{\prime}}\right)\left(r y^{\prime}\right)\right| d y^{\prime} \\
& =(1-r)^{-1}\|f\|_{B_{0}} \int_{\mathbb{S}}\left|\Lambda_{m+1} \nabla_{y}^{-1}\left(g * \nabla_{y} P_{x^{\prime}}\right)\left(r y^{\prime}\right)\right| d y^{\prime} \\
& =(1-r)^{-1}\|f\|_{B_{0}} \int_{\mathbb{S}}\left|\Lambda_{m+1}\left(g * P_{x^{\prime}}\right)\left(r y^{\prime}\right)\right| d y^{\prime} \\
& \leq \tilde{N}_{1}(g)\|f\|_{B_{0}}(1-r)^{-m-2}, \quad r \in I, \quad x \in \mathbb{B} .
\end{aligned}
$$

Therefore $M_{\infty}\left(\Lambda_{m+1} \nabla h, r^{2}\right) \leq C(1-r)^{-m-2}$ for all $r \in I$. This implies (see [4], Chapter 7) that $M_{\infty}\left(\nabla h, r^{2}\right) \leq C(1-r)^{-1}$. This proves that $M_{c}$ maps $B_{0}$ into $B$. Since harmonic polynomials are dense in $B_{0}$ (see [6]), and $M_{c}$ maps harmonic polynomials into harmonic polynomials it follows that $M_{c}$ maps $B_{0}$ into $B_{0}$.

The following proposition is a partial extension of Theorem 7 .

Proposition 9. Let $0<p \leq 1, p \leq q \leq \infty$ and $m>\alpha-1$. If a double indexed sequence $c=\left\{c_{k}^{j}: k \geq 0,1 \leq j \leq d_{k}\right\}$ satisfies the following condition:

$$
N_{1}(g)=\sup _{0 \leq \rho<1} \sup _{y^{\prime} \in \mathbb{S}}(1-\rho)^{m+1-\alpha+\beta} \int_{\mathbb{S}}\left|\Lambda_{m+1}\left(g * P_{x^{\prime}}\right)\left(\rho y^{\prime}\right)\right| d x^{\prime}<\infty,
$$

where $g(x)=\sum_{k \geq 0} r^{k} \sum_{j=1}^{d_{k}} c_{k}^{j} Y_{j}^{(k)}\left(x^{\prime}\right)$, then $c \in M_{H}\left(B_{\alpha}^{p, \infty}, B_{\beta}^{q, \infty}\right)$. 
Proof. Let us assume that condition (36) is satisfied. Let $f \in B_{\alpha}^{p, \infty}(\mathbb{B})$ and set $h=c * f$. We have, using Lemma 6 :

$$
\begin{aligned}
h\left(r \rho x^{\prime}\right) & =\int_{\mathbb{S}}\left(g * P_{x^{\prime}}\right)\left(r y^{\prime}\right) f\left(\rho y^{\prime}\right) d y^{\prime} \\
& =2 \int_{0}^{1} \int_{\mathbb{S}} \Lambda_{m+1}\left(g * P_{x^{\prime}}\right)(r R \xi) f(\rho R \xi)\left(1-R^{2}\right)^{m} R^{n-1} d \xi d R .
\end{aligned}
$$

Using (37) and $M_{\infty}(f, \rho R) \leq M_{\infty}(f, R)$ we obtain, for $x=r x^{\prime} \in \mathbb{B}$ :

$$
\left|h\left(r \rho x^{\prime}\right)\right| \leq 2 \int_{0}^{1} \int_{\mathbb{S}}\left|\Lambda_{m+1}\left(g * P_{x^{\prime}}\right)(r R \xi)\right| M_{\infty}(f, R)\left(1-R^{2}\right)^{m} R^{n-1} d \xi d R .
$$

Now letting $\rho \rightarrow 1$ and using condition (36) we obtain

$$
\begin{aligned}
\left|h\left(r x^{\prime}\right)\right| & \leq 2 \int_{0}^{1} M_{\infty}(f, R)\left(1-R^{2}\right)^{m} R^{n-1} \int_{\mathbb{S}}\left|\Lambda_{m+1}\left(g * P_{x^{\prime}}\right)(r R \xi)\right| d \xi d R \\
& \leq 2^{m+1} \int_{0}^{1} M_{\infty}(f, R)(1-R)^{m} R^{n-1} \int_{\mathbb{S}}\left|\Lambda_{m+1}\left(g * P_{\xi}\right)\left(r R x^{\prime}\right)\right| d \xi d R \\
& \leq 2^{m+1} N_{1}(g) \int_{0}^{1} M_{\infty}(f, R)(1-R)^{m} R^{n-1}(1-r R)^{\alpha-\beta-m-1} d R
\end{aligned}
$$

Since $M_{\infty}(f, R)$ is an increasing function we can apply Lemma 3 from [3] to obtain

$$
\left|h\left(r x^{\prime}\right)\right|^{p} \leq C \int_{0}^{1} M_{\infty}^{p}(f, R) \frac{(1-R)^{m p+p-1}}{(1-r R)^{p(m+1+\beta-\alpha)}} R^{n-1} d R .
$$

This estimate is valid for all $x^{\prime} \in \mathbb{B}$ and therefore gives estimate for $M_{\infty}^{p}(h, r)$ which is used, together with Lemma 2, in the following inequalities:

$$
\begin{aligned}
& \|h\|_{B_{\beta}^{p, \infty}}^{p} \\
= & \int_{0}^{1} M_{\infty}^{p}(h, r)(1-r)^{\beta p-1} r^{n-1} d r \\
\leq & C \int_{0}^{1} M_{\infty}^{p}(f, R)(1-R)^{m p+p-1} R^{n-1} \int_{0}^{1} \frac{(1-r)^{\beta p-1}}{(1-r R)^{p(m+1+\beta-\alpha)}} r^{n-1} d r d R \\
\leq & C \int_{0}^{1} M_{\infty}^{p}(f, R)(1-R)^{\alpha p-1} R^{n-1} d R=C\|f\|_{B_{\alpha}^{p, \infty}}^{p} .
\end{aligned}
$$

Since, by (12), $\|h\|_{B_{\beta}^{q, \infty}} \leq C\|h\|_{B_{\beta}^{p, \infty}}$ for $p \leq q$, the proof is complete.

Our last theorem shows that restriction $1 \leq p$ in Theorem 6 can be removed in the case $s=\infty$.

Theorem 9. Let $0<p \leq q \leq \infty$ and $m>\alpha-1$. Then for a double indexed sequence $c=\left\{c_{k}^{j}: k \geq 0, \overline{1} \leq \bar{j} \leq d_{k}\right\}$ the following conditions are equivalent:

1. $c \in M_{H}\left(B_{\alpha}^{p, 1}, B_{\beta}^{q, \infty}\right)$. 
2. The function $g(x)=\sum_{k \geq 0} r^{k} \sum_{j=1}^{d_{k}} c_{k}^{j} Y_{j}^{(k)}\left(x^{\prime}\right)$ is harmonic in $\mathbb{B}$ and satisfies the following condition

$$
N_{\infty}(g)<\infty .
$$

Proof. The case $1 \leq p \leq \infty$ was settled in Theorem 6 . Assume $0<p<1$, the necessity of the condition $N_{\infty}(g)<\infty$ was established in Lemma 7. Now we assume $g=g_{c}$ satisfies $N_{\infty}(g)<\infty$ and we use the same method of proof as in Proposition 9. Let $f \in B_{\alpha}^{p, 1}$ and set $h=M_{c} f$. Starting from (37) and using $M_{1}(f, \rho R) \leq M_{1}(f, R)$ we obtain

$$
\left|h\left(r \rho x^{\prime}\right)\right| \leq 2 \int_{0}^{1}\left(1-R^{2}\right)^{m} R^{n-1} M_{1}(f, R) \sup _{\xi, x^{\prime} \in \mathbb{S}}\left|\Lambda_{m+1}\left(g * P_{x^{\prime}}\right)(r R \xi)\right| d R .
$$

Then, analogously to the proof of Proposition 9 , we let $\rho \rightarrow 1$ to obtain, using condition (38):

$$
\left|h\left(r x^{\prime}\right)\right| \leq 2^{m+1} N_{\infty}(g) \int_{0}^{1} M_{1}(f, R)(1-R)^{m}(1-r R)^{\alpha-\beta-m-1} R^{n-1} d R .
$$

Since $M_{1}(f, R)$ is an increasing function, one can follow the same reasoning as in the proof of Proposition 9, replacing $M_{\infty}(f, R)$ by $M_{1}(f, R)$, to obtain $\|h\|_{B_{\beta}^{p, \infty}} \leq C\|f\|_{B_{\alpha}^{p, 1}}$. That suffices, due to embedding (12).

\section{References}

[1] A. B. Alexandrov, Essays on non Locally Convex Hardy Classes, in Complex Analysis and Spectral Theory, 1-89, Lecture Notes in Mathematics 864, 1981.

[2] — On decrease in mean at the boundary of harmonic functions, in Russian, Algebra i Analiz, Tom 7 (1995), no. 4, 1-49.

[3] M. Arsenović and R. F. Shamoyan, Sharp theorems on multipliers and distances in harmonic function spaces in higher dimension, J. Siberian Federal University. Mathematics \& Physics 5 (2012), no. 3, 291-302.

[4] M. Djrbashian and F. Shamoian, Topics in the theory of $A_{\alpha}^{p}$ classes, Teubner Texts in Mathematics, 105. BSB B. G. Teubner Verlagsgesellschaft, Leipzig, 1988.

[5] M. Jevtić and M. Pavlović, Harmonic Bergman functions on the unit ball in $\mathbb{R}^{n}$, Acta Math. Hungar. 85 (1999), no. 1-2, 81-96.

[6] Harmonic Besov spaces on the unit ball in $\mathbb{R}^{n}$, Rocky Mountain J. Math. 31 (2001), no. 4, 1305-1316.

[7] J. M. Ortega and J. Fabrega, Holomorphic Triebel-Lizorkin Spaces, J. Funct. Anal. 151 (1997), no. 1, 177-212

[8] M. Pavlović, Convolution in the harmonic Hardy space $h^{p}$ with $0<p<1$, Proc. Amer. Math. Soc. 109 (1990), no. 1, 129-134.

[9] Multipliers of the vanishing Hardy classes, Publ. Inst. Math. (Beograd) (N.S.) 52(66) (1992), 34-36.

[10] R. F. Shamoyan, On multipliers from Bergman type to Hardy spaces in polydisk, Ukrain. Mat. Zh. 52 (2000), no. 10, 1405-1414; translation in Ukrainian Math. J. 52 (2000), no. 10, 1606-1617.

[11] _ Holomorphic Lizorkin Triebel type spaces in the unit polydisk, Izv. NAN Armenii 3 (2002), 57-78.

[12] R. F. Shamoyan and A. Abkar, On multipliers of spaces of harmonic functions in the unit ball of $\mathbb{R}^{n}$, J. Inequalities and Special Functions 3 (2012), no. 1, 1-9. 
[13] A. Shields and D. Williams, Bounded projections, duality and multipliers in spaces of harmonic functions, J. Reine Angew. Math. 299/300 (1978), 256-279.

[14] E. M. Stein and G. Weiss, Introduction to Fourier Analysis on Euclidean Spaces, Princeton University Press, 1971.

[15] M. Zakaryan, Integral representations and duality in weighted spaces of harmonic functions in the unit ball, in Russian, Ph.D. Thesis, Erevan State University, 1999.

Miloš Arsenović

Department of Mathematics

UNIVERSITY OF BELGRADE

Studentski Trg 16, 11000 Belgrade, Serbia

E-mail address: arsenovic@matf.bg.ac.rs

Romi F. SHAMOYAN

Department of Mathematics

BRYANSK UNIVERSITY

Bryansk, Russia

E-mail address: rshamoyan@yahoo.com 